Archives

$12 \mid 1994$

Polysémie de la santé. Institutions et pratiques sociales en France et au Québec 1750-1980

\title{
L'introduction de l'enseignement de l'hygiène au Québec
}

\section{Georges Desrosiers}

\section{(2) OpenEdition \\ 12 Journals}

\section{Édition électronique}

URL : http://journals.openedition.org/ccrh/2743

DOI : $10.4000 /$ ccrh. 2743

ISSN : 1760-7906

Éditeur

Centre de recherches historiques - EHESS

Édition imprimée

Date de publication : 12 avril 1994

ISSN : 0990-9141

Référence électronique

Georges Desrosiers, "L'introduction de l'enseignement de l'hygiène au Québec », Les Cahiers du Centre de Recherches Historiques [En ligne], 12 | 1994, mis en ligne le 27 février 2009, consulté le 07 mai 2019. URL : http://journals.openedition.org/ccrh/2743; DOI : 10.4000/ccrh.2743

Ce document a été généré automatiquement le 7 mai 2019.

Article L.111-1 du Code de la propriété intellectuelle. 


\title{
L'introduction de l'enseignement de l'hygiène au Québec
}

\author{
Georges Desrosiers
}

Au Québec, bien que les trois premières facultés de médecine aient été créées dans la première moitié du XIX ${ }^{e}$ siècle, l'enseignement de l'hygiène n'a été inclus dans le cursus des études médicales que beaucoup plus tardivement. L'étude dont nous présentons les résultats confirme qu'il ne suffit pas qu'il y ait un savoir disponible pour qu'une matière appartenant au champ de la médecine soit reconnue officiellement et enseignée dans les facultés de médecine. Il faut qu'un certain nombre de médecins parmi l'élite de la profession, dont l'influence pése sur les décisions, soient convaincus que la possession de ce savoir particulier par les étudiants en médecine répond à un besoin social et que la profession y trouve son intérêt. Il faut, de plus, que ce groupe ait la motivation et le dynamisme nécessaires, dans un contexte socio-politique favorable, pour entraîner une partie de la profession et convaincre les décideurs politiques à qui revient, finalement, la responsabilité d'édicter les conditions d'admission

des candidats à l'exercice de la médecine. Ces conditions ne furent pas réunies au Québec avant les années 1870. 


\section{Introduction}

1 Il y a au Québec quatre facultés de médecine rattachées aux universités de Sherbrooke, McGill, Montréal et Laval. La Faculté de médecine de l'Université de Sherbrooke n'ayant été créée qu'en 1966 n'est pas incluse dans cette étude ${ }^{1}$. Les trois premières facultés ou, dans certains cas, les écoles qui les ont précédées ont été créées dans la première moitié du $19^{\mathrm{e}}$ siècle : la "Montreal Medical Institution" qui a précédé la Faculté de médecine de l'Université McGill en 1823 ; l'École de médecine et de chirurgie de Montréal en 1843 qui deviendra la Faculté de médecine de l'Université de Montréal en 1920 et l'École de médecine de Québec en 1848 qui cédera la place à la Faculté de médecine de l'Université Laval de Québec en 1854. En 1871, s'ajoute une quatrième faculté, la Faculté de médecine de l'Université Bishop's qui sera intégrée à la Faculté de médecine de l'Université McGill en 1905. Au départ, les programmes des trois premières facultés ou écoles n'offraient aucun enseignement d'hygiène, pas même à l'intérieur d'un autre cours. Ce n'est que plus tardivement, dans la deuxième moitié du siècle que l'hygiène est apparue comme matière d'enseignement obligatoire. L'Université Laval de Québec a fait oeuvre de pionnière en créant une chaire d'hygiène dès 1857. Par la suite, lorsque l'Université Bishop's a créé sa propre faculté de médecine à Montréal en 1871, elle incluait une chaire pour l'enseignement obligatoire de l'hygiène. Par contre, les deux autres facultés attendirent que le Collège des médecins et chirurgiens de la province de Québec impose l'enseignement de l'hygiène comme matière obligatoire pour la délivrance du permis d'exercice en 1876 pour s'y conformer. Cette étude couvre la période qui va de 1823 à 1878, année où toutes les facultés offraient un enseignement obligatoire de l'hygiène.

2 Dès la fin du XVIII ${ }^{e}$ siècle, en France et dans les pays allemands, l'hygiène était considérée comme un corps de connaissance suffisamment organisé et son utilité suffisamment reconnue pour être incluse comme matière obligatoire dans le cursus des études médicales. À cette époque les pays germaniques mirent en avant le concept de police médicale, c'est-à-dire l'établissement d'une politique sanitaire par le gouvernement et son implantation par voie administrative. On tenta d'appliquer le concept à tous les grands problèmes de santé2. L'œuvre de Frank, contenue dans son traité sur un système complet de police médicale en six volumes publiés entre 1779 et 1817 , présente un système complet et détaillé d'hygiène publique et individuelle qui prévoyait la prise en charge des sujets par le souverain de la naissance à la mort. C'est en 1786 à Pavie dans la partie de l'Italie sous la domination autrichienne que Johann Peter Frank, qui occupait le poste de titulaire de la chaire de médecine clinique de l'Université et de directeur de la santé publique pour la Lombardie autrichienne, introduisit un enseignement de l'hygiène. Par la suite, cette initiative de Frank fut imitée dans les autres villes des pays germaniques ${ }^{3}$.

Le fondement de la création des premières chaires d'hygiène dans les écoles de médecine françaises repose sur les écrits des philosophes du XVIII ${ }^{\mathrm{e}}$ siècle inspirés par la croyance en la perfectibilité de l'homme par l'éducation et des institutions libres. L'humanisme de ces penseurs s'est exprimé dans la monumentale Encyclopédie de Diderot publiée entre 1751 et 1772 qui visait à améliorer la condition humaine. Dans le domaine de la santé publique, les auteurs ont traité entre autres de la durée de la vie, de l'arithmétique politique, de l'homme, de la population, des hôpitaux, des enfants trouvés. Diderot s'est intéressé particulièrement au problème de la mortalité infantile et a insisté sur le devoir du 
souverain de prendre des mesures pour la réduire ${ }^{4}$. Il prévoit le développement de l'hygiène qui prolongera la durée moyenne de la vie. Ces idées furent reprises par les membres de la Société royale de médecine qui tentèrent d'en formuler l'application pratique. La Révolution de 1789 a permis par l'abolition des anciennes structures et la création de nouvelles, de faire passer les idées des philosophes. Toutes les corporations enseignantes sont supprimées en 1792, et en 1794 la Convention crée trois écoles de médecine à Paris, Montpellier et Strasbourg pour remplacer les anciennes facultés de médecine. Douze chaires à plein temps sont instituées dont une chaire de physique médicale et d'hygiène ${ }^{5}$. Au Royaume-Uni, Andrew Duncan d'Édimbourg qui donna à partir de 1795 des conférences hebdomadaires sur le droit médical, sous l'influence de Franck, consacra une partie de son enseignement à l'hygiène. Il recommanda la création d'une chaire dans cette discipline. Le premier poste de professeur fut créé en 1807. Mais les progrès furent lents puisqu'en 1856. Rumsey déplorait le fait qu'il n'y ait encore aucun professeur à plein temps dans les universités. Édimbourg a été la première université britannique à créer une chaire d'hygiène à plein temps en $1898^{6}$.

4 Le premier enseignement d'hygiène dans une faculté de médecine d'Amérique du Nord a été donné en 1835 à l'Université du Maryland. Mais c'était l'exception, il faudra attendre jusqu'à 1910 pour que l'École de médecine de l'Université Harvard engage un professeur de médecine préventive à plein temps dans la personne du célèbre professeur Rosenau?

Autant en France que dans les pays germaniques, l'enseignement de l'hygiène constituait une partie d'un ensemble de mesures permettant à l'Etat central d'assurer la surveillance de la santé de la population par des médecins habilités à la faire. Ce courant n'eut que peu d'influence au Royaume-Uni et dans les autres pays anglo-saxons comme les États-Unis et le Canada où l'État n'avait pas tendance à réglementer la vie privée des sujets du roi ou des citoyens selon le cas. De plus, la responsabilité de l'hygiène publique incombait traditionnellement aux municipalités, ce qui excluait pratiquement toute possibilité d'établir une véritable politique nationale en matière sanitaire. Il y a bien eu des initiatives ponctuelles qui, cependant, n'eurent pas de suites pratiques comme, par exemple, le programme de santé publique présenté par le docteur Tessier dans le Journal de Québec en 1826-18278.

6 Notre hypothèse est qu'il ne suffit pas qu'il y ait un savoir disponible pour qu'une matière appartenant au champ de la médecine soit reconnue officiellement et soit enseignée dans les facultés de médecine. Il faut qu'un certain nombre de médecins parmi l'élite de la profession, dont l'influence est appelée à peser sur les décisions, soient convaincus que la possession de ce savoir particulier par les étudiants en médecine réponde à un besoin social et que la profession y trouve son intérêt. Il faut, de plus, que ce groupe ait la motivation et le dynamisme nécessaire, dans le cadre d'un contexte socio-politique favorable, pour entraîner une partie de la profession et convaincre les décideurs politiques à qui revient, finalement, la responsabilité d'édicter les lois qui déterminent les conditions d'admission des candidats à l'exercice de la médecine. Ces conditions ne furent pas réunies au Québec avant les années 1870.

7 Nous allons d'abord présenter le contexte socio-politique et démographique du BasCanada à l'époque de la création des premières écoles ou facultés de médecine pour ensuite décrire les circonstances de la création de chacune des quatre écoles et les étapes de l'introduction de l'enseignement de l'hygiène. Finalement, nous ferons l'analyse des différents facteurs qui ont influencé le processus qui a conduit à un enseignement 
obligatoire. Nous réservons l'analyse détaillée du contenu même de l'enseignement de l'hygiène pour une étude ultérieure.

\section{Le contexte socio-politique et démographique}

8 En 1823, au moment de la création de la première école de médecine, le Québec est une colonie britannique peuplée d'une majorité de Canadiens (francophones) et d'une minorité de loyalistes et d'immigrants britanniques qui détiennent le pouvoir économique. Il existe depuis 1792 une Assemblée législative formée de députés élus par la population dans laquelle les Canadiens sont majoritaires. Le pouvoir exécutif est concentré dans les mains du gouverneur nommé par le gouvernement britannique, s'appuyant sur un conseil dont les membres, majoritairement anglophones, sont nommés par le gouvernement. La bourgeoisie locale naissante est engagée dans une lutte politique dont l'enjeu est la suprématie de l'Assemblée législative. Plusieurs médecins participent activement à cette lutte 9 .

9 Les soixante ans de régime anglais l'ont imprégné de l'esprit des institutions britanniques pénétrées de la notion de non-intervention de l'Etat dans la vie privée des sujets du roi. C'est pourquoi le gouvernement ne s'intéressait que peu au champ de la santé, si ce n'est que pour appliquer la quarantaine dans les périodes d'épidémie et réglementer de façon très large l'accès à la pratique de la médecine par le moyen de ce qu'on appelait un bureau d'examinateurs nommés par le gouverneur. À partir des années 1830, il confie aux municipalités nouvellement créées la responsabilité du génie sanitaire et de l'hygiène publique selon la tradition britannique ${ }^{10}$.

En 1847, sous la pression des médecins qui avaient accru leur pouvoir politique à l'occasion des luttes pour la suprématie de l'Assemblée, est créé le Collège des médecins et chirurgiens du Bas-Canada, organisme parapublic administré par un «bureau de gouverneurs » chargé de veiller à l'application de la loi adoptée régissant la profession médicale, notamment les conditions d'admission aux études médicales, les conditions d'octroi du permis d'exercice de la médecine incluant le contenu des études médicales, la déontologie et la répression de l'exercice illégal de la médecine.

11 Un article de la loi précisait les cours que devait suivre obligatoirement le candidat au permis d'exercice. Ce sont: l'anatomie, la physiologie, la physique, la chimie, la botanique, la matière médicale (thérapeutique), la clinique médicale, chirurgicale et obstétricale. Il n'était pas question d'hygiène.

12 Cependant, si cette loi confiait beaucoup de responsabilités au Collège, elle ne lui accordait que peu de pouvoir pour les assumer, ce qui incitera les médecins à revenir à la charge dans les années 1870. Cette limitation venait plus de l'opposition de la Faculté de médecine de l'Université McGill à l'octroi de tels pouvoirs que d'une volonté de l'État de limiter les pouvoirs de la profession médicale. Les médecins de McGill y voyaient une intrusion des praticiens, surtout francophones, dans les prérogatives des établissements d'enseignement médical ${ }^{11}$.

13 En 1820, il n'y a aucun établissement d'enseignement supérieur au Canada. L'enseignement n'est pas réglementé. Pour pouvoir ouvrir un établissement, il suffit que quelques notables adressent une requête au Gouverneur dans laquelle ils précisent l'objet de leur demande. S'ils sont bien appuyés politiquement, ils obtiennent une charte qui constitue une reconnaissance officielle. Ils doivent s'autofinancer, bien qu'ils puissent 
recevoir quelques subventions du gouvernement, non nécessairement récurrentes. C'est de cette manière que les quatre écoles de médecine qui font l'objet de cette étude ont été créées.

14 Au début du siècle les personnes qui se destinaient à l'exercice de la médecine se formaient par apprentissage auprès d'un praticien notoire, puisqu'il n'y avait pas d'écoles. Une petite minorité qui en avait les moyens allait se former à l'étranger, généralement à New York, à Paris, à Londres ou à Édimbourg. Quelques praticiens donnaient des leçons privées d'anatomie, de physiologie ou de chimie à ces apprentis. Ce sont ces premiers enseignants privés qui se sont regroupés pour fonder les premières écoles de médecine. Ces écoles se finançaient principalement en imposant aux étudiants des frais pour chaque cours suivi. Dans ces conditions, les cours libres n'étaient pas très courus $^{12}$.

En 1831, le Québec est peu peuplé, mais sa population double en quarante ans, passant de 553000 habitants à 1200000 en 1871. On note une importante différence dans l'évolution de la population des deux villes de Québec et de Montréal. La population de Québec double, celle de Montréal fait plus que tripler. Montréal s'industrialise beaucoup plus tôt et plus intensément que Québec. C'est d'abord dans cette ville que naîtra et se développera le mouvement hygiéniste.

Tableau 1

Évolution de la population ${ }^{13}$

\begin{tabular}{c|c|c}
\hline & 1831 & 1871 \\
\hline $\begin{array}{c}\text { Bais-Canedda } \\
\text { (Province de Quebec) }\end{array}$ & 553130 & 1191500 \\
Québec (ville) & 27741 & 59670 \\
Montreall & 31800 & 107700
\end{tabular}

\section{La faculté de médecine de l'université McGill}

Quatre médecins attachés à l'Hôpital général de Montréal fondèrent en 1823 la "Montreal Medical Institution" qui commença à dispenser les cours aux étudiants en octobre 1824. Les matières enseignées étaient l'anatomie et la physiologie, la chimie et la pharmacie, l'obstétrique et les maladies des femmes, la matière médicale, la botanique, la chirurgie et la physique pratique. En 1828, cette école devenait la Faculté de médecine de l'Université McGill appelée à l'époque McGill College. En 1844, s'ajoute un cours de médecine légale. Mais il faudra attendre 1857 pour que quelques leçons dites de police médicale soient dispensées par le professeur R.P. Howard à l'intérieur du cours de médecine légale dont il prenait charge. En 1860, la description du cours de médecine légale indique qu'on traite aussi d'hygiène publique et de police médicale. De 1862 à 1869, le cours de médecine légale est attribué au docteur McCallum, un autre professeur de clinique médicale. À partir de 1862 les manuels recommandés apparaissent dans l'annuaire. Pour le cours de médecine légale on recommande la Médecine légale d'Orfila, la Médecine légale théorique et pratique de Devergie, Taylor's Jurisprudence, Guy's Forensic Medicine, Taylor on Poisons. De 1869 à 1875 c'est le docteur Fendwick, un professeur de clinique chirurgicale qui donne le cours de médecine légale avec le même contenu que son prédécesseur. 
17 C'est en 1875 qu'on crée la première chaire d'hygiène qui est confiée au docteur Robert Godfrey qui de 1871 à cette date avait enseigné l'obstétrique et la chirurgie à la Faculté de médecine de l'Université Bishop's. Cette annonce faisait dire au directeur de la revue "Public Health Magazine" dans le numéro de septembre 1875 :

We can most heartily congratulate the student of medicine on this event, because it is most sincerely to be hoped, and reasonably expected, that the object of the Faculty in the creation of the Chair is to make the subject no more an optional summer course, attended by few, if any, but one to be delivered at a season when a full attendance during one of the matriculation years may be put in by each student, and the course made so full and instructive as to become thoroughly popular and appreciated. The fact that the calendar is silent, both as to the time when the lectures shall be delivered, or even the fees to be paid for attendance, looks very like the old hum-drum system that prevailed in our own undergraduate days. It is certain that the community expect a well-qualified medical man to be some authority on the subject and practice of Hygiene, and as it is a daily topic of inquiry, and an all-important matter in town and country, let our deservedly popular University sustain its character in this branch as in all others...

18 Le cours ne deviendra obligatoire qu'en 1878-79. On mentionne dans l'annuaire qu'il est d'une durée de trois mois et pour la première fois on ajoute l'hygiène à la liste des matières dont les manuels sont recommandés, soit Parks, Hammond et Wilson. De 1880 à 1883, le cours est donné par le docteur Gardner, professeur de médecine légale. Dans l'annuaire de 1882 on fournit pour la première fois la liste des sujets traités : l'eau de boisson et l'adduction en eau; les conditions du sol et de l'eau qui affectent la santé, comme le drainage et les différentes méthodes de traitement des ordures ; l'atmosphère, incluant la chaleur et la ventilation ; l'hygiène individuelle comprenant des sujets comme l'alimentation et les boissons; l'exercice physique et les bains; les associations de citoyens pour la promotion de l'hygiène ${ }^{14}$.

\section{École de médecine et de chirurgie de Montréal}

Cette École est mise sur pied en 1843 par un groupe de cinq médecins anglophones de Montréal en riposte au monopole exercé par la Faculté de médecine de l'Université McGill. De bilingue qu'elle était au début elle deviendra complètement française à partir de 1849. Dans la Loi autorisant sa création, il est mentionné que les matières à enseigner sont: l'anatomie et la physiologie, la chimie et la pharmacie, la matière médicale, la théorie et la pratique de la médecine, les principes et la pratique de la chirurgie et de l'obstétrique et les maladies des femmes et des enfants ${ }^{15}$. Dans les années qui suivent on ajoute d'autres cours, notamment un cours de médecine légale en $1850^{16}$. En 1874, le docteur Georges Grenier, jeune démonstrateur d'anatomie depuis quelques années est chargé de donner quelques leçons d'hygiène ${ }^{17}$. La première chaire d'hygiène sera créée en 1876 et confiée au docteur Emmanuel Persillier Lachapelle. Mais ce n'est qu'un cours libre qui deviendra obligatoire l'année suivante en 1877-78.

Dans l'annuaire de cette année scolaire on annonce le cours comme suit :

Le cours d'hygiène, dont l'importance et la nécessité sont universellement reconnus est aujourd'hui obligatoire.

Ce cours a pour objet: l'application des lois sanitaires à la santé publique; à prescrire les mesures hygiéniques propre à prévenir et combattre les maladies contagieuses; à répandre les connaissances hygiéniques que chaque médecin doit posséder.

Les sujets suivants seront traités: modificateurs physiques: chaleur, lumière, électricité, son, pesanteur ; modificateurs chimiques : air, eau, sol, aliments ; modificateurs biologiques 
et individuels: sexe, âge, hérédité, constitution, tempérament, etc.; modificateurs

sociologiques : profession, famille, nationalité.

21

$$
\begin{aligned}
& \text { description en ajoutant : } \\
& \text { En traitant chaque agent ou modificateur, on fera connaitre: } \\
& \text { 1. ses caractères physiques, ses propriétés; } \\
& \text { 2. son action sur l'organisme; } \\
& \text { 3. les maladies qu'il peut provoquer; } \\
& \text { 4. les règles d'hygiène privée et publique qui en découlent }{ }^{18} \text {. }
\end{aligned}
$$
est essentiellement théorique. Dans l'annuaire de 1878-1879, on reproduit la même

\section{L'école de médecine de Québec et la faculté de médecine de l'université Laval}

L'École de médecine de Québec fondée en 1845 par un groupe de douze médecins de Québec n'ouvrira cependant ses portes qu'en 1848. Le cursus des études médicales correspond exactement à la description qui vient d'être faite pour l'École de médecine et de chirurgie de Montréal. L'enseignement de l'hygiène n'apparaît pas au programme. L'École ferme ses portes en 1854, cédant la place à la Faculté de médecine de l'Université Laval.

L'inauguration de la nouvelle faculté est présidée le 25 septembre 1854 par le doyen Jean Blanchet qui brosse un tableau des sciences médicales de son temps et des diverses matières qui seront enseignées par les premiers professeurs. Il n'est aucunement question d'hygiène dans ce discours ${ }^{19}$.

En 1855, la direction de la faculté envoie un tout jeune diplômé, le docteur Hubert Larue se former en chimie et en médecine légale à Louvain puis à Paris. Lorsqu'il revient d'Europe durant l'été 1857, il est nommé secrétaire de la faculté et professeur extraordinaire titulaire de la chaire de médecine légale créée quelques années plus tôt et d'une chaire d'hygiène. Le mystère reste complet sur les circonstances de la création de cette nouvelle chaire ${ }^{20}$. En effet, dans la correspondance régulière que le docteur Larue a entretenue avec les autorités de l'Université durant son stage en Europe il est souvent question de chimie et un peu de toxicologie mais nous n'avons pu retrouver aucune allusion à l'hygiène. On ne peut qu'émettre des hypothèses. Il est possible qu'il ait pris contact avec le professeur Bouchardat titulaire de la chaire d'hygiène de l'École de médecine de Paris dans les années 1850 et le docteur Michel Lévy auteur du Traité d'hygiène publique et privée paru pour la première fois en 1844. Il fut titulaire de la chaire d'hygiène jusqu'en $1882^{21}$. Comme les annuaires de l'Université Laval du XIX ${ }^{e}$ siècle ne fournissent aucune description des cours ou des manuels recommandés, il est difficile de retracer le contenu de son enseignement. Cependant, et heureusement, un de ses élèves qui a suivi ses cours de médecine légale et d'hygiène durant l'année 1872, les a copiés mot à mot, et ayant légué ses notes de cours à l'Université, elles permettent de reconstituer le contenu de l'enseignement du professeur Larue. On retrouve dans ces notes exactement la même division que celle du traité d'hygiène publique et privée de Michel Lévy, ainsi que la même terminologie ${ }^{22}$. 


\title{
La faculté de médecine de l'université Bishop's
}

En 1871 s'ouvre à Montréal une troisième école de médecine affiliée à l'Université Bishop's. Contrairement aux autres écoles cependant, l'hygiène fait partie des matières obligatoires dont l'enseignement est confié au professeur Jean Leprohon.

Le contenu du cours est décrit comme suit :

Enseigner l'application des sciences de l'hygiène aux questions de santé publique.

Enseigner les mesures hygiéniques à prendre pour la prévention et l'arrêt des maladies contagieuses et fournir des connaissances sur les principes de la médecine d'état qui peuvent être utiles pour le médecin praticien.

Les sujets suivants seront traités :

l'alimentation, l'eau, la ventilation, les systèmes de ramassage des ordures, leur influence sur le taux de mortalité dans les villes, les statistiques médicales, les prisons et les écoles de réforme, la pauvreté, le crime et la folie, les effets du climat sur la santé.

Comme manuels, on recommande le Traité d'hygiène publique et privée de Michel Lévy et le Practical Hygiene de Parkes ${ }^{23}$.

\section{La situation à la fin de la période}

En 1877-78, les quatre facultés de médecine se conformaient aux exigences de la nouvelle loi adoptée en décembre 1876 par l'Assemblée législative de la province de Québec et intitulée « Acte pour amender et refondre les actes concernant la profession médicale et la chirurgie dans la province de Québec » dont l'article 14 qui portait sur le contenu des études médicales était rédigé comme suit :

\begin{abstract}
Les qualifications requises de tout candidat qui se présentera à l'examen pour obtenir une licence afin d'être autorisé à pratiquer seront qu'il ait atteint l'âge de vingt-et-un ans; qu'il ait étudié sans interruption pendant une période de pas moins de quatre années à dater de son admission à l'étude de la médecine par le bureau; et qu'il ait suivi, pendant les dites quatre années, dans quelque université, collège ou école de médecine incorporée, dans les domaines de Sa Majesté, pas moins de deux cours de six mois chacun, d'anatomie générale ou descriptive, - d'anatomie pratique, - de chirurgie, - de pratique de la médecine, - de l'art obstétrique,-de chimie,-de matière médicale et thérapeutique générale,-des institutes (sic) de médecine ou de physiologie et de pathologie générale, - de clinique médicale et - de clinique chirurgicale, - un cours de six mois ou deux cours de trois mois de jurisprudence médicale, - et un cours de botanique de trois mois, - un cours d'hygiène de trois mois... ${ }^{24}$
\end{abstract}

L'hygiène était élevée au rang des matières dont la connaissance est dorénavant obligatoire pour pouvoir exercer la médecine.

On note que les effectifs professoraux et étudiants sont dispersés en de petites unités comme le montre le tableau suivant.

Tableau 2 


\begin{tabular}{c|c||c} 
& 1852 & 1874 \\
\hline MC Gill & 64 & 129 \\
EMICM & 24 & 99 \\
EMIQ" & 16 & 93 \\
Bishlop's & - & 30
\end{tabular}

\section{Le processus qui a conduit à l'enseignement obligatoire de l'hygiène}

Comment en est-on arrivé à cette décision ? C'est ce que nous allons maintenant retracer. Deux événements de nature tout à fait différente, n'ayant à première vue aucun lien entre eux, sont intervenus qui ont permis de réunir les conditions permettant d'adopter une législation qui rendait l'enseignement de l'hygiène obligatoire dans les facultés de médecine. Il s'agit d'abord de la naissance et du développement du mouvement hygéniste à Montréal auquel ont adhéré plusieurs médecins. L'autre événement a été la fondation de l'Association médicale canadienne en 1867 dont l'objectif prioritaire était la création d'un Bureau central d'examinateurs pour l'ensemble du Canada, chargé de faire subir un examen à tous les candidats à l'exercice de la médecine.

Au début des années 1860, Montréal est une ville en pleine expansion industrielle et dont la population approche le cap des 100000 habitants. Les conditions de vie de la classe ouvrière sont misérables. Plusieurs contemporains en ont donné une description dont les docteur Adolphe Dagenais et Lemire en 1865, alors qu'ils étaient rédacteurs de la Gazette médicale :

Nous voyons souvent jusqu'à quatre et cinq familles logées dans des ruelles, des cours, où le sol est toujours humide, et fangeux, où la lumière ne donne que sur l'entrée de la maison, et

l'air impossible d'être renouvelé a cause du défaut d'ouvertures nécessaires. Ajoutons maintenant que ces familles se composent quelques fois de six enfants et plus, et l'on ne sera pas surpris du développement excessif des maladies et de la fréquence des mortalités... ${ }^{26}$

Le premier deviendra à partir de 1872 professeur d'obstétrique à l'École de médecine et de chirurgie de Montréal ; le second était membre du Bureau de santé de la ville.

Les conditions de salubrité et d'hygiène sont parmi les pires en Amérique du Nord comme le révélaient le docteur J.G. Bibaud et Philip Carpenter dans le rapport annuel de la «Montreal Sanitary Association» qu'ils rédigeaient en mars 1867: «To this body (the Board of Health) was entrusted the work of cleaning a city confessedly one of the most filthy on the continent, of which the mortality was frighfully in excess of the country districts round ${ }^{27}$.»

La variole fait des ravages. Cette situation provoque une forte réaction au sein de la classe bourgeoise qui se sent menacée dans sa qualité de vie par les déplorables conditions de salubrité et qui craint pour sa santé devant l'expansion de la variole. On se mobilise. La santé et la salubrité deviennent un enjeu politique à l'échelon de l'administration municipale compte tenu du fait que ces questions étaient du ressort des villes dans le Canada de cette époque. 
C'est dans ce contexte qu'en mars 1866, un groupe de notables montréalais majoritairement anglophones, inspirés par les initiatives prises dans certaines grandes villes américaines notamment dans la ville de New York, fondent la « Montreal Sanitary Association ", connue sous le nom d'Association sanitaire dans les milieux francophones. En plus de lutter pour la salubrité, elle prendra résolument partie pour la vaccination obligatoire contre la variole. Ses buts tels que décrits dans le premier rapport annuel en mars 1867 sont: "Generally, to collect and diffuse information, and take action on all matters relating to the Public Health; and specially, to assist in improving the abodes of the poorer classes ${ }^{28}$."

Son premier président, William Workman, fort de l'appui de l'association, qui se fera élire maire de la ville en 1868 , était porteur d'un programme réformiste en matière de salubrité et de santé publique. Un certain nombre de médecins feront partie de l'association comme Jean-Philippe Rottot, professeur de médecine légale à l'École de médecine et de chirurgie, qui jouera un rôle déterminant dans l'adoption de la loi de 1876, A.B. Laroque, premier officier de santé de la cité à plein temps, R.P. Howard, premier professeur d'hygiène à McGill, Jean Leprohon qui deviendra en 1871 le premier professeur d'hygiène à la Faculté de médecine de l'Université Bishop's, William Hingston, chirurgien à l'Hôtel-Dieu, professeur à l'École de médecine et de chirurgie et futur maire de Montréal en 1875, J.G. Bibaud, professeur d'anatomie à l'École de médecine et de chirurgie de Montréal, G.E. Fendwick et G.W. Campbell, professeurs de chirurgie à McGill et ce dernier doyen de la facultée 29 .

37 À partir de 1865, l'administration de la ville commence à accorder une place plus importante aux médecins dans l'administration de l'hygiène publique. En vertu des nouveaux règlements, on crée un Bureau de santé qui se compose du Comité de santé déjà existant formé exclusivement d'échevins et de onze médecins dont au moins six sont professeurs à McGill et à l'École de médecine et de chirurgie, parmi lesquels figura le président de cette dernière, le docteur Pierre Munro ${ }^{30}$.

Dans les années 1870, l'hygiène publique continue d'être un enjeu majeur de la politique montréalaise. En 1875, le docteur William Hingston est élu maire. Il compte faire avancer la cause de la salubrité et de l'hygiène publique de trois façons : par l'obtention de la part du Gouvernement de la Province de pouvoirs juridiques et législatifs plus étendus, par la réorganisation de l'administration de la santé publique, notamment en renforçant la présence médicale, enfin par une vigoureuse campagne antivariolique ${ }^{31}$.

La « Montreal Sanitary Association », qui a été active jusqu'en 1874, est remplacée en 1875 par une autre association qui porte le nom de «Citizens' Public Health Association ». En effet le 15 mai 1875, à une assemblée publique convoquée par Hingston nouvellement élu maire de Montréal pour rendre compte de l'état sanitaire de la ville et faire état des mesures à prendre, il est résolu «qu'une association sanitaire de citoyens soit formée dans le but de prêter appui aux autorités constituées, et d'enseigner au peuple tout ce qui se rattache aux réformes sanitaires ${ }^{32} »$. Le docteur George Baynes élu premier secrétaire s'exprimait ainsi à la réunion de fondation :

Montreal is the most unhealthy city in the Dominion and yet we live on never thinking that we are responsible for its condition... The question of the sanitary health of the city is a very difficult one, but not so difficult as it was some years ago, when sanitary science was in its infancy and was not so well understood. We have here now some of the most able medical men this side of the Atlantic. 
40

urs médecins rejoignirent les rangs de cette nouvelle association dont C.O. Perrault et Georges Grenier qui comme on le verra ont joué un rôle décisif dans la prise de décision de la jeune Société médicale de Montréal (1872), en ce qui concerne l'organisation de l'enseignement de la médecine ${ }^{33}$.

41 Le militantisme des médecins engagés dans le mouvement hygiéniste se manifeste dans plusieurs secteurs, notamment dans le journalisme médical. Par exemple la Gazette médicale, revue mensuelle médico-chirurgicale dirigée par Adolphe Dagenais et E. Lemire, consacre en 1865-66, une partie importante de ses pages aux questions d'hygiène publique. L'Union médicale du Canada fondée en 1872, est une autre revue médicochirurgicale qui, sous la direction de Rottot, Grenier et Dagenais, continuera l'action de la Gazette médicale. Le "Public Health Magazine », organe officiel de la "Citizen's Public Health Association » de 1875 à 1877, dirigée par le docteur George Baynes, est la première revue médicale canadienne consacrée exclusivement à l'hygiène. Les rédacteurs utilisent largement les pages de leurs revues pour revendiquer un rôle plus important pour les médecins en hygiène publique et pour faire valoir la nécessité d'un enseignement de l'hygiène aux étudiants en médecine.

En 1865, le rédacteur de La Gazette médicale écrivait dans un article sur le Comité de santé :

Personne n'est à même comme le médecin de connaître les besoins hygiéniques d'une localité. Par ses études qui l'obligent à s'occuper d'hygiène, par ses occupations qui l'appellent dans tous les endroits de la ville, depuis les lieux choisis par la classe riche jusqu'aux lieux occupés par la classe pauvre, par ses relations qui le mettent en rapport avec les personnages les plus élevés de la société et avec les êtres les plus bas dans l'échelle sociale, le médecin est sans contredit l'homme qui peut rendre le plus de services quand il s'agit de l'assainissement d'une ville... ${ }^{34}$

A. Dagenais écrivait dans l'Union médicale en juin 1872 au sujet de la composition du Bureau de santé de la ville :

Pour faire des lois et des règlements sur l'hygiène publique et les faire exécuter, il faut des connaissances spéciales qui ne s'acquièrent que par de fortes études. On peut être bon notaire, un avocat habile, un marchand consommé dans les affaires, et cependant, ignorer une foule de choses qui se rattachent à l'état sanitaire d'une ville ${ }^{35}$.

44 En juillet 1874, le rédacteur en chef de l'Union médicale à l'occasion d'un vibrant plaidoyer en faveur d'un renforcement des pouvoirs du Collège des médecins sur l'enseignement médical, déclarait :

Si nous examinons maintenant le programme d'études médicales prescrit par la loi actuelle, nous constatons qu'il est assez complet pour permettre à celui qui s'en est rendu maître, d'exercer la médecine avec fruit. Afin de le rendre plus parfait, on devrait cependant exiger des cours particuliers sur certaines branches telles que l'hygiène... ${ }^{36}$

À la suite de l'adoption de la loi de 1876, le rédacteur du «Public Health Magazine » déclarait: "What please us most as Sanitarians, is, that the very point we have been advocating for months and months, that a compulsory course of Hygiene, is at last required for qualification to examination ${ }^{37}$. "

46

La fondation de l'Association médicale canadienne à Québec en 1867 sera le point de départ d'une lutte qui conduira à la loi de décembre 1876. En effet, dès la réunion de fondation de cette association, il fut proposé et accepté, comme un de ses principaux objectifs, la création d'un Bureau national d'examinateurs par une loi du parlement fédéral visant à uniformiser, à l'échelle de tout le Canada, les conditions d'admission à l'exercice de la médecine. Les tenants de cet important déplacement du pouvoir de réglementer la profession médicale de l'échelon provincial à l'échelon fédéral mirent 
plusieurs années à élaborer leur projet puisque ce n'est qu'en 1872 qu'il fut soumis à l'approbation de ses membres. Rottot, ardent partisan de l'autonomie provinciale, prit la tête de l'opposition à ce projet à l'intérieur et à l'extérieur de l'association. Il afficha la couleur, pour ainsi dire, dès le premier numéro de l'Union médicale en janvier 1872 et n'eut de cesse que de voir adopter la loi de décembre 1876.

À la réunion de l'Association médicale canadienne (convoquée le 11 septembre 1872) spécialement destinée à adopter le projet qu'on appellait dans l'Union médicale «l'acte médical projeté", Rottot, appuyé par une grand nombre de membres québécois francophones et fort du mouvement de protestation populaire qu'il avait initié, déposa une motion de rejet du projet qui fut acceptée après un débat houleux ${ }^{38}$. Le danger de l'intrusion fédérale dans un champ de compétence provinciale était écarté, mais encore fallait-il combler le vide créé par une initiative au niveau provincial. Il fut d'autant plus facile de mobiliser les médecins qu'il y avait un fort courant de mécontentement devant la faiblesse des mesures que pouvait prendre le Collège pour combattre la concurrence jugée déloyale des charlatans et réglementer les conditions d'accès aux études et à l'exercice de la médecine qu'on trouvait trop laxistes et qui avaient comme résultat de produire une pléthore de médecins. Un autre événement milita en faveur de cette cause, ce fut l'adoption par l'Assemblée législative de l'Ontario, durant cette même année 1872, d'une loi conférant au collège des médecins de cette province le pouvoir de faire subir un examen d'admission à l'exercice de la médecine à tout candidat, y compris les diplômés des facultés de médecine de la province.

Au Québec, le mouvement en faveur de l'amendement à la loi médicale ne prit pas racine au niveau du collège, contrairement à ce à qu'on aurait pu raisonnablement attendre. Le Collège n'avait pas réussi depuis sa fondation en 1847 à surmonter l'espèce d'inertie dans laquelle il était enlisé à cause d'un conflit interne entre les gouverneurs issus du milieu universitaire, principalement ceux de l'Université McGill et à un moindre degré de l'Université Laval, et les autres sur les rôles respectifs des universités et du collège concernant l'admission en médecine, le contenu, et la valeur probante du diplôme universitaire pour le droit d'exercice. McGill tenait jalousement à ses prérogatives et s'opposait farouchement à toute réduction de celles-ci, utilisant tous les moyens à sa disposition pour empêcher ou retarder toute décision du Bureau des gouverneurs à l'encontre de celles-ci.

C'est par le biais de la jeune et dynamique Société médicale de Montréal que s'enclencha le processus de révision de la loi médicale. En août 1872, sur une proposition de Rottot, l'assemblée rejette le projet de l'Association médicale canadienne et nomme un comité restreint de trois membres qui militaient tous dans le mouvement hygiéniste, Rottot qui le préside, Grenier et Perreault, chargés de préparer les amendements nécessaires ${ }^{39}$. Le 5 septembre, le comité dépose son rapport qui recommande de rejeter définitivement le projet de l'Association médicale canadienne et soumet un certain nombre de propositions devant servir de base à un projet d'amendement à la loi sur le Collège des médecins et chirurgiens de la Province de Québec.

Parmi ces propositions, apparaît pour la première fois l'exigence d'un cours obligatoire d'hygiène pour pouvoir se présenter à l'examen :

Que personne ne pourra se présenter pour obtenir la licence du Collège, s'il n'a suivi durant quatre années consécutives, (à commencer depuis la date de son admission à l'étude la médecine) dans une université, collège ou école de médecine canadienne incorporée: deux cours de six mois l'Anatomie et Physiologie; do(sic) d'Anatomie pratique; do de Chirurgie; 
do de Théorie et pratique de médecine; do l'Accouchements, maladie des femmes et des enfants; do de Chimie; do de Matière médicale et pharmacie. Un cours de 6 mois d'Institutes (sic) de Médecine; un cours de 2 mois de Jurisprudence médicale et Toxicologie; un cours de 3 mois de Botanique ; 6 mois de Clinique médicale; 6 mois de Clinique chirurgicale ; 3 mois de Maternité ou un certificat prouvant qu'il a eu 5 cas d'accouchements; un cours de 3 mois d'Hygiène; do de Chimie pratique; do de Chirurgie pratique ${ }^{40}$.

51 En novembre 1875, trois ans après l'adoption de ce projet, le Bureau des Gouverneurs du Collège n'ayant pas réussi malgré les pressions de l'intérieur et de l'extérieur à s'entendre sur les amendements à apporter à la loi, la Société médicale de Montréal délègue le docteur E.-P. Lachapelle auprès du Gouvernement pour le presser de déposer à l'Assemblée législative un projet d'amendement à la loi médicale répondant à ses attentes ${ }^{41}$. La requête de la Société est bien reçue et un projet de loi est déposé en première lecture dès le 25 novembre ${ }^{42}$. À l'annonce de ce coup de force, le Bureau des Gouverneurs du Collège, qui se voyait court-circuité crie au scandale. Cependant il se met enfin à étudier sérieusement le projet et au cours des séances du 8 mai et du 27 septembre 1876, il entérine des propositions formulées par les gouverneurs Howard et Rottot, tous deux engagés depuis longtemps dans le mouvement hygiéniste, qui rendent le projet du Collège identique à celui de la Sociétét ${ }^{43}$. Et finalement, le 22 décembre l'e législative adopte la loi.

\section{Conclusion}

Lorsqu'elle a décidé d'introduire un enseignement de l'hygiène, la Faculté de médecine de l'Université Laval n'a pas été influencée par le mouvement hygiéniste qui ne s'est manifesté dans la ville de Québec que dans les années $1890^{44}$. Par ailleurs, jusqu'à ce que le docteur Hubert Larue revienne de Paris en 1857 où il avait été envoyé pour se former en chimie et en médecine légale et non en hygiène, ni l'École de médecine de Québec, ni la faculté qui lui a succédé n'avaient manifesté un intérêt quelconque pour l'hygiène entre 1848 et 1857. Cela nous conduit à conclure que, dans cette faculté, c'est la force de persuasion d'un individu brillant qui a permis d'introduire un enseignement obligatoire de l'hygiène bien avant les facultés montréalaises.

À Montréal la situation s'est présentée de façon tout à fait différente. Comme on l'a montré, le mouvement hygiéniste a été très fort dès les années 1860, au point d'influencer de façon décisive la politique sanitaire de l'administration de la ville en faisant de l'hygiène publique un enjeu politique majeur. Dès la naissance du mouvement, plusieurs médecins parmi les plus en vue, notamment des enseignants des deux facultés, s'y sont engagés et ont même occupé des postes dans les structures nouvelles qui ont été mises en place. De nouvelles revues médicales ont vu le jour qui, comme l'Union médicale et le "Public Health Magazine », ont appuyé systématiquement le mouvement. Il y avait un climat favorable dans le milieu médical qu'on n'avait jamais connu auparavant. Ainsi que le mentionnaient des leaders d'opinion comme Rottot, Dagenais, Grenier et Baynes, il s'ouvrait pour les médecins un nouveau champ de pratique qui ne pouvait être que le bienvenu à une époque où plusieurs se plaignaient de l'encombrement de la profession. Aussi insistaient-ils sur la compétence naturelle des médecins pour traiter de ces questions, ce qui justifiait le fait de leur laisser occuper le champ. Mais encore fallait-il que le discours soit concordant avec la réalité. C'est pourquoi, ils soulignaient l'urgence de rendre l'enseignement de l'hygiène obligatoire dans le cursus des études médicales. Les progrès étaient particulièrement lents puisque, si la jeune Faculté de Bishop's créait 
une chaire dès l'ouverture des cours en 1871, McGill se contentait d'un cours libre en 1875, de même que l'École de médecine et de chirurgie en 1876.

C'est vraiment la lutte de pouvoir qui s'engagea entre les médecins canadiens-français du Québec et les médecins canadiens-anglais au sujet du rôle respectif des instances nationales et provinciales quant à la réglementation de l'admission à l'exercice de la médecine entre 1867 et 1872, puis ensuite entre les partisans et les adversaires d'un rôle accru du collège des médecins et chirurgiens concernant les études médicales et les conditions d'admission à l'exercice de la médecine, qui fut l'occasion pour les hygiénistes de faire avancer leur cause. Étant particulièrement bien placés, à cause du rôle que certains d'entre eux ont joué dans cette lutte, ils ont su habilement faire passer leurs propositions sans soulever de débats au moment des prises de décisions importantes. La loi, adoptée par le parlement provincial, engageait automatiquement toutes les universités sans exception.

\section{NOTES}

1. Roger Maltais, «Le Centre médical de l'Université de Sherbrooke », Sherbrooke, les Editions de l'Université de Sherbrooke, 1980, p. 316. L'inauguration des cours a eu lieu le 6 septembre 1966.

2. George Rosen, A History of Public Health, New York : MD Publ., 1976, p. 161-7.

3. Henry Sigerist, Landmarks in the History of Hygiene, New York : Oxford UP, 1956, p. 47-63. George Rosen, op. cit. note 2, p. 131-4.

4. A. Colieu, Centenaire de la Faculté de médecine de Paris (1794-1894), Paris : Imprimerie nationale, 1896, p. 1.

5. A. Colieu, Centenaire de la Faculté de médecine de Paris (1794-1894), Paris : Imprimerie nationale, 1896, p. 1.

6. James M. Mackintosh, "Teaching and Practice of Preventive Medicine", The Lancet, (juillet 1944), p. 6305 ; F. Grundy et J. M. Mackintosh, L'enseignement de l'hygiène et de la santé publique en Europe, Genève : OMS, 1950, p. 23-5.

7. Wilson G. Smillie, Public Health. Its Promise and Future, New York : Macmillan, 1955, p. 15, 235-6, 442-5.

8. Othmar Keel et Peter Keating, «Autour du journal de médecine de Québec. Programme scientifique et programme de médicalisation », in R.A. Jarrell et N. Ball (dir), Critical Issues in the History of Canada Science, Technology and Medicine, Ottawa : HSTC Public, 1983, p. 101-35.

9. André Garon, «Le Bas-Canada (1792-1838)», in Jean Hamelin (dir), L'histoire du Québec, Montréal : Éd. France-Amérique, 1977, p. 283-343 ; Fernand Ouellet, Histoire économique et sociale du Québec 1760-1850, Montréal : Fides, 1966.

10. Denis Goulet et André Paradis, Trois siècles d'histoire médicale au Québec, Montréal : VLB, 1992, p. 208-14.

11. Loi créant le collège des médecins et chirurgiens du Bas-Canada, Québec: The Consolidated Statute for Lower Canada, 1860. Jacques Bernier, «Vers un nouvel ordre médical : les origines de la Corporation des médecins et chirurgiens du Québec », Recherches sociographiques, XXII. 3, (1981), p. 307-30. 
12. Jacques Bernier, La médecine au Québec, Québec: Les Presses de l'Université Laval, 1989, p. 31-4.

13. Y. Bernier et R. Boily, Le Québec en chiffres de 1850 à nos jours, Montréal : Acfas, p. 25 et 44.

14. Annuaires de la Faculté de médecine de l'Université McGill, Montréal: Archives de l'Université, 1854-5 à 1885-6. Maud Abbott, An Historical Sketch of the Medical Faculty of McGill University, Montréal : Bibliothèque Osler, Université McGill, Reproduit du Montreal Medical Journal, août 1902. Francis J. Shepperd, "The First Medical School in Canada, its History and Founders ", The Canadian Medical Association Journal, XV (1925), p. 83-84.

15. Denis Goulet, op. cit. note 9, p. 19-22.

16. Procès-verbaux du Conseil de l'École de médecine et de chirurgie de Montréal, Montréal : Archives de l'Université de Montréal, 1845-60.

17. «Université Victoria », L'Union médicale du Canada, III. 12 (1874), p. 576.

18. Annuaires de l'École de médecine et de chirurgie de Montréal, Montréal : Archives de l'Université de Montréal, 1860-80.

19. Charles M. Boissonneault, Histoire de la Faculté de médecine de l'Université Laval, Québec: Les Presses de l'Université Laval, 1953, p. 135-43 et 167-79. "Medical Faculty of Laval University ", The Medical Chronical, II. 3 (1854), p. 167, présente la liste des enseignants et des enseignements pour $1853:$ l'hygiène n'y apparaît pas.

20. Procès-verbaux du Conseil de la Faculté de médecine de l'Université Laval, Québec: Archives de l'Université Laval, 1856-84.

21. Charles M. Boissonneault, op. cit. note 18, p. 194-204. Annuaires de la Faculté de médecine de l'Université Laval, Québec: Archives de l'Université Laval, 1857-84. Hubert Larue, Correspondance, Québec: Archives de l'Université Laval, 1855-7. Correspondance pendant son séjour en Europe.

22. O. Mazurette, Hygiène privée par le docteur Hubert Larue, Québec, Fonds O. Mazurette du Service d'archives de l'Université Laval, 13 avril 1872. Il s'agit de ses notes de cours d'étudiant en médecine. Michel Lévy, Traité d'hygiène publique et privée, Paris : Baillière, 1844.

23. Annuaires de la Faculté de médecine de l'Université Bishop's, Montréal : Archives de l'Université McGill, 1871-82.

24. Statuts de la Province de Québec, chapitre 26, Québec : 1876, p. 81-7.

25. Francis J. Shepperd., op. cit. note 13, p. 422 pour l'année 1852, et L'Union médicale du Canda, III. 9 et 10 (1874), p. 429 et 476 pour la première session de 1874.

26. A. Dagenais et E. Lemire, «Hygiène publique », Gazette médicale, I. 3 (1865), p. 33-4.

27. J. G. Bibaud et P. Carpenter, First Annual Report of the Montreal Sanitary Association 28 mars 1867. Reproduit du Montreal Herald, Archives de l'Université du Québec à Montréal ( ${ }^{\circ}$ 36), p. 1.

28. Ibid., p. 1.

29. Ibid., p. 4.

30. J. G. Bibaud, op. cit. note 26, p. 1.

31. Michael Farley, Othmar Keel, Camille Limoges, «Les commencements de l'administration montréalaise de la santé publique (1865-1885)», in R. Jarrell et James Hull (dir), Science, Technology and Medicine in Canada's Past, Thornhill : The Scientia Press Ltd, 1991, p. 279. À ce jour, cette étude est la plus complète sur l'administration de la santé publique à Montréal pour cette période.

32. "Question sanitaire ", L'Union médicale du Canada, IV. 6 (1875), p. 287-8.

33. George Baynes, «Sanitary Reports ", Public Health Magazine, I. 1 (1875), p. 6-7.

34. A. Dagenais et E. Lemire, «Le Comité de Santé », La Gazette médicale, I. 2 (1865), p. 17.

35. A. Dagenais, « Du Bureau de Santé », L'Union médicale du Canada, I. 6 (1872), p. 252-3.

36. "Amendements à l'acte d'incorporation de la profession médicale de la Province de Québec », L'Union médicale du Canada, (juillet 1874), p. 324. 
37. "The Act of Amendment Relating to the Practice of Medicine in the Province of Quebec ", Public Health Magazine, II. 2 (1877), p. 254.

38. J.P. Rottot, «L'Association médicale canadienne ", L'Union médicale du Canada, I. 10 (1872), p. 437-45.

39. «Société médicale de Montréal (séance du 7 août 1872) », L'Union médicale du Canada, I, 9, (1872), p. 410.

40. «Société médicale de Montréal (séance du 5 septembre 1872) », L'Union médicale du Canada, I. 10 (1872), p. 458-62.

41. «Société médicale de Montréal (séance du 17 novembre 1875) », L'Union médicale du Canada, V. 1 (1876), p. 15.

42. «Acte concernant la Profession Médicale de la Province de Québec », L'Union médicale du Canada, IV. 12 (1875), p. 554-74.

43. Collège des médecins et chirurgiens de la Province de Québec, Procès-verbaux 1871-1882, no 3, Montréal : Archives de la Corporation des médecins du Québec, 10 mai et 27 septembre 1876.

44. Réjean Lemoine, «La santé publique : de l'inertie municipale à l'offensive hygiéniste », in G.H. Dagneau (dir.), La ville de Québec, histoire municipale. IV - De la Confédération à la crise de 1929, Québec : La Société historique de Québec, 1983, p. 31-180.

\section{AUTEUR}

\section{GEORGES DESROSIERS}

Professeur au département de médecine sociale et préventive, université de Montréal 